\title{
Microbial composition in Hyalomma anatolicum collected from livestock in the United Arab Emirates using next-generation sequencing
}

Nighat Perveen, Sabir Bin Muzaffar, Ranjit Vijayan and Mohammad Ali Al-Deeb* ${ }^{*}$

\begin{abstract}
Background: Hyalomma anatolicum is a widely distributed tick species that acts as a vector transmitting tick-borne pathogens (TBPs) in livestock. Such pathogens affect the health of livestock and consequently reduce their productivity. Knowledge about the microbial communities (pathogens and endosymbionts) of ticks in the United Arab Emirates (UAE) is scarce. Therefore, the aim of the present study was to quantify microbial diversity in $\mathrm{H}$. anatolicum using next-generation sequencing (NGS) technology.
\end{abstract}

Methods: Hyalomma anatolicum ticks were collected from livestock in the emirates of Abu Dhabi, Dubai and Sharjah in the UAE during 2019. DNA was extracted from 175 male ticks sampled from livestock $(n=78)$ and subjected to NGS. The 165 rRNA gene was analyzed using the Illumina MiSeq platform to determine the bacterial communities. Principal coordinates analysis (PCA) was performed to identify patterns of diversity in the bacterial communities.

Results: Twenty-six bacterial families with high relative abundance were identified, of which the most common were Staphylococcaceae, Francisellaceae, Corynebacteriaceae, Enterobacteriaceae, Moraxellaceae, Bacillaceae, Halomonadaceae, Xanthomonadaceae, Pseudomonadaceae, Enterococcaceae, Actinomycetaceae and Streptococcaceae. The diversity of the microbial communities in terms of richness and evenness was different at the three study locations, with the PCA showing clear clusters separating the microbial communities in ticks collected at Abu Dhabi, Dubai, and Sharjah. The presence of bacterial families harboring pathogenic genera showed that $\mathrm{H}$. anatolicum could pose a potential threat to livestock and food security in the UAE.

Conclusions: The study is the first to document important data on the microbial communities associated with $\mathrm{H}$. anatolicum in the UAE. This knowledge will facilitate a better understanding of the distribution pattern of microbes in livestock ticks in the UAE and, ultimately, will aid in deciphering the relationships between microbes and in the exploration of potential factors towards developing effective management strategies.

Keywords: Hyalomma anatolicum, 165 rRNA gene, Microbial communities, Pathogens, Livestock

*Correspondence: m_aldeeb@uaeu.ac.ae

Biology Department, College of Science, United Arab Emirates University, P.O. Box 15551, Al-Ain, United Arab Emirates

\section{Background}

In many areas of the world, tick-borne diseases and tick-related health problems cause significant morbidity in both animals and people. Ticks (Acari: Ixodida) are obligate hematophagous ectoparasites of vertebrates, including humans [1]. They pose a serious threat to the original author(s) and the source, provide a link to the Creative Commons licence, and indicate if changes were made. The images or other third party material in this article are included in the article's Creative Commons licence, unless indicated otherwise in a credit line to the material. If material is not included in the article's Creative Commons licence and your intended use is not permitted by statutory regulation or exceeds the permitted use, you will need to obtain permission directly from the copyright holder. To view a copy of this licence, visit http://creativecommons.org/licenses/by/4.0/. The Creative Commons Public Domain Dedication waiver (http://creativeco mmons.org/publicdomain/zero/1.0/) applies to the data made available in this article, unless otherwise stated in a credit line to the data. 
livestock industry by adversely affecting the host's health through irritation and inflammation on feeding sites, skin damage, anemia, toxicosis and paralysis, or by transmitting a variety of pathogens that cause tick-borne diseases $[1,2]$. The ongoing climate change is also a major factor contributing to the spread of tick-borne pathogens in animals and humans across various geographic areas and the emergence or re-emergence of tick-borne diseases [3]. Hyalomma anatolicum, a widely distributed tick species that occurs worldwide, is the main vector of piroplasmids, including Theileria annulata, T. lestoquardi, T. equi and Babesia caballi, as well as Crimean-Congo hemorrhagic fever virus [4]. In a recent study, $H$. anatolicum in the United Arab Emirates (UAE) was found to be infected with T. annulata and Theileria ovis [5].

The varied assemblages of pathogenic and non-pathogenic microbes inhabiting the tick gut has effects on the microbes themselves as well as on the host. Ticks harbor a diverse community of microorganisms that could affect vector competence, i.e. the ability of a vector to successfully transmit a pathogen $[6,7]$. The interactions between bacterial pathogens and symbionts in tick microbiota remain unresolved. Assemblages of microbes could negatively or positively influence each other, resulting in the suppression or enhancement of some microbial species $[7,8]$. In addition, the tick microbiome probably affects the colonization of pathogens within the tick vector and, subsequently, their transmission to hosts [9], possibly altering the epidemiology of tick-borne diseases. For example, removal of the midgut bacteria of Ixodes scapularis feeding on antibiotic-treated mice impairs infection by Borrelia burgdorferi [10]. The presence of endosymbiotic Rickettsia bellii in Dermacentor andersoni is associated with lowered infection rates of pathogenic Anaplasma marginale [11]. Also, a decrease in Francisella endosymbionts is associated with lower Francisella novicida infection levels in Dermacentor andersoni [11]. Such inter-species interactions suggest that endosymbionts may play an essential role in manipulating the transmission of tick-borne pathogens through effects on microbial community structure $[12,13]$. It is still unclear if ticks depend on bacterial endosymbionts to provide vitamins and cofactors that are lacking in the vertebrate host's blood [14]. However, when Coxiella-like bacterium detected in Amblyomma americanum ticks $[15,16]$ were eliminated with antibiotics, there was a severe reduction in tick fecundity and fitness was observed that corresponded with an increase in host fitness [17]. Genomic analysis of the $A$. americanum microbiome communities revealed the metabolic interdependencies of ticks and their nutrient-provisioning endosymbionts, suggesting their potential role as nutritional mutualists [14]. Thus, understanding microbial interactions and their functions at the community level is of vital importance to a better understanding of tick-borne pathogen transmission to animals or humans [9]

Numerous studies have been carried out on the microbiome of ticks infesting livestock $[6,18,19]$. In the UAE, ticks are a continuous problem in livestock [5], particularly in camels. In a study conducted in 2019-2020, the camel tick, Hyalomma dromedarii, was found on camels throughout the year [20]. However, to date, only one study has been conducted in the UAE on the bacterial genera present in $H$. dromedarii [8], indicating a lack of research on the microbiomes of several important tick vectors in the country. The microbiomes of ticks could play important roles in the maintenance of tick populations on livestock under unfavorable environmental conditions. Overall, little research has been conducted to quantify tick microbial communities in the UAE. Therefore, the aim of the present study was to quantify microbial diversity in $H$. anatolicum using next-generation sequencing (NGS) technologies. The results will strengthen our understanding of both tick-microbe and microbe-microbe relationships.

\section{Methods}

\section{Tick collection and identification}

The current study had a cross-sectional design. A total of 994 ticks were collected from cows (22), goats (20) and sheep (36) between March and July 2019 from the emirates of Abu Dhabi, Dubai and Sharjah in the UAE. Ticks were collected in sterile $50-\mathrm{ml}$ tubes, placed in an icebox and transported to the Animal Ecology and Entomology Laboratory, UAE University, Al-Ain. Once in the laboratory, the ticks were stored at $-80{ }^{\circ} \mathrm{C}$ until DNA extraction. The identification of $H$. anatolicum ticks was confirmed morphologically using taxonomic keys [21, 22].

Tick collection was carried out in strict accordance with the recommendations of the Animal Research Ethics Committee (A-REC) of UAE University (ethical approval no.: ERA_2019_5953). The experimental protocol was also approved by the UAE University Research Office.

\section{Genomic DNA extraction and pooling}

Only male $H$. anatolicum were selected for DNA extraction due to an insufficient number of female ticks. Before DNA extraction, ticks were washed in 70\% ethanol and deionized water for 5 min to remove environmental contaminants in accordance with a published protocol [23]. Due to the small size of each tick, a pool of five male $H$. anatolicum ticks was homogenized in liquid nitrogen inside a sterile $1.5-\mathrm{ml}$ microcentrifuge tube using a sterile Kimble Kontes pellet pestle (Thermo Fisher Scientific, Waltham, MA, USA). Genomic DNA was extracted from 
each pool using the QIAamp Tissue Kit (Qiagen, Hilden, Germany) following the manufacturer's protocol. The concentration and quality of DNA were assessed using a Nano Drop ND-100 spectrophotometer (Peqlab Biotechnologie $\mathrm{GmbH}$, Erlangen, Germany). DNA quality was also determined by electrophoresis in a $1 \%$ agarose gel; the bands were stained with ethidium bromide and visualized under UV light. DNA was stored at $-20{ }^{\circ} \mathrm{C}$ in the freezer until further use. Prior to sequencing, extracted DNA samples (5 DNA samples of ticks from each host) were pooled again to make one pool for each host from each location, resulting in seven DNA pools.

\section{Next-generation sequencing and bioinformatics analysis}

$16 S$ ribosomal RNA (rRNA) gene-based analysis was conducted to determine the composition of the bacterial communities in $H$. anatolicum. For NGS, each of the seven DNA pools were sequenced by Macrogen Inc. (Seoul, South Korea). A pair of primers, Bakt_341F (CCT ACG GGNGGC WGC AG) and Bakt_805R (GAC TAC HVGGG TAT CTA ATC C [24]), was used to amplify the hypervariable $\mathrm{V} 3-\mathrm{V} 4$ region of the $16 S$ rRNA gene. PCR was performed using the Herculase II Fusion DNA polymerase Nextera XT Index Kit V2 (Agilent Technologies, Inc., Santa Clara, CA, USA), and sequencing was performed on an Illumina MiSeq platform (Illumina, Inc., San Diego, CA, USA) with a read length of $301 \mathrm{bp}$. Fast length adjustment of short reads (FLASH) version 1.2.11 [25] was used to merge FASTQ pairedend sequences. Read quality by sample is given in Additional file 1: Table S1. Next, merged reads were clustered into operational taxonomic units (OTUs) using CDHIT-OTU [26] with default options. The CD-HIT-OTU workflow includes several preprocessing steps and filters low-quality reads, trims long tails, identifies chimeric reads and, finally, clusters the reads into OTUs with an identity cutoff of $97 \%$. Finally, the assign_taxony.py script from QIIME1.9.1 [27] was used for the taxonomic assignment of OTUs. The assignment was based on Basic Local Alignment Search Tool (BLAST) [28] searches in the National Center for Biotechnology Information (NCBI) $16 S$ microbial database and the Ribosomal Database Project (RDP; http://rdp.cme.msu.edu/). Taxonomic abundance count was aggregated in Microsoft Excel (Microsoft Corp., Redmond, WA, USA) to calculate abundance ratios at the phylum, class, family and genus levels.

\section{Statistical analyses}

Principal Coordinates Analysis (PCoA) was conducted to determine diversity patterns in bacterial communities in $H$. anatolicum using the Paleontological Statistics Software Package (PAST) 5.27 [29]; the OTU count for each genus was entered and the samples were classified by locations (Abu Dhabi, Dubai, and Sharjah). We examined the Eigenvalues to determine the magnitude of variation [30]. Different indices of diversity were calculated since a single index often does not reflect the true nature of diversity and a combination provides a better approximation of diversity. Richness (total number of genera, based on OTUs obtained for each genus), the Shannon-Wiener Index and the Index of Dominance were estimated. Pearson's correlation coefficient $(r)$ was calculated to determine the associations between different genera [31]. Stepwise regression analysis was performed with backward selection with the genera that only showed significant correlations [31]. The value of $\alpha$ was set at 0.05 for all tests.

\section{Results}

\section{Bacterial 165 rRNA gene abundance profile}

A total of 476,949 read counts were obtained (range $51,680-83,201$ reads, average $68,135.571$ reads). The raw reads were subsequently used for taxonomic classification after the sequences were subjected to quality filtering. A total of 372 OTUs were generated through the de novo picking process (clustered at $97 \%$ similarity), belonging to 14 phyla, 22 classes, 81 families and 153 genera.

\section{Relative abundance of bacteria}

Taxonomic profiling of the microorgansms collected from $H$. anatolicum resulted in the identification of eight phyla that were abundant: Proteobacteria, Firmicutes, Actinobacteria, Bacteroidetes, Fusobacteria, Cynobacteria, Planctomycetes and Chloroflexi. Of these Proteobacteria and Firmicutes were the most abundant phyla, and Chloroflexi and Planctomycetes were the least abundant (Additional file 2: Table S2).

Ten bacterial classes of the 22 identified were abundant: Gammaproteobacteria, Bacilli, Actinobacteria, Clostridia, Alphaproteobacteria, Betaproteobacteria, Fusobacteriia, Flavobacteriia, Erysipelotrichia and Bacteroidia. Gammaproteobacteria was recorded as the most dominant class in ticks collected from cows (from Dubai), goats (from Sharjah), and sheep (from Abu Dhabi), while Bacilli was found to be the most dominant class in ticks collected from sheep from Dubai and Sharjah, cows from Sharjah and goats from Dubai (Additional file 3: Table S3).

Taxonomic assignment revealed that 26 bacterial families were abundant (Additional file 3: Table S3), among which the following showed the highest relative abundance: Francisellaceae (72\%) detected in ticks collected from goats, Enterobacteriaceae (57.9\%) and Moraxellaceae (38.8\%) detected in ticks from cows and 
Staphylococcaceae (57.7\%), Corynebacteriaceae (41.5\%), Halomonadaceae (31.7\%) and Bacillaceae (23.6\%) detected in ticks from sheep (Fig. 1; Additional file 4: Table S4).

In the microbiome of $H$. anatolicum, the relative abundance of genera was highly variable among all hosts. Staphylococcus and Corynebacterium were the two most common genera reported from all locations, with high relative abundance detected in sheep (57.6\% and $41.5 \%$, respectively). Francisella had a relative abundance of $72 \%$ in ticks collected from goats, followed by Proteus (57.9\%) from cows and Carnimonas (31.7\%) and Bacillus (23.6\%) from sheep. Acinetobacter, Psychrobacter, Ignatzschineria, Streptococcus, Pseudomonas, Massilia, Klebsiella, Trueperella and Enterococcus occurred at moderately low relative abundances (6-22\%). However, the genera Brevibacterium, Fusobacterium, Enterobacter, Peptoniphilus, Nesterenkonia, Peptostreptococcus, Wautersiella, Murdochiella, Clostridium, Alloiococcus, Turicella, Arthrobacter, Propionibacterium, Helcococcus, Sporosarcina, Parvimonas, Anaerococcus, Salmonella, Enhydrobacter and Auritidibacter were all found to be present at low relative abundance (1-5.4\%) (Fig. 2; Additional file 5: Table S5).

\section{Microbial richness and evenness}

Principal coordinates analysis showed that coordinates 1 , 2,3 and 4 accounted for $>84 \%$ of the variation (based on cumulative Eigenvalues), with the first two coordinates accounting for $>54 \%$ of the variation. There was also a clear separation of the microbial communities according to location (Fig. 3). The richness of genera differed significantly between cow samples from Dubai and Sharjah (4.24 in cows, Dubai [C.D] versus 1.56 in cows, Sharjah [C.S]; two-sample paired t-test, $P<0.01)$. The ShannonWiener index differed significantly between C.D and C.S (1.118 [95\% confidence interval: 1.111-1.127] vs 1.308 [95\% confidence interval: 1.302-1.315], respectively; two sample paired t-test, $P<0.05)$. The Index of Evenness was significantly lower in C.D than in C.S ( 0.25 vs 0.37 , respectively; two-sample paired t-test, $t=-35.747$, $P<0.01)$. The Index of Dominance was also significantly different between C.D and C.S (0.45 vs 0.33 , respectively; two-sample paired t-test, $P<0.01)$. Similarly, the richness of genera differed significantly between goat samples from Dubai and Sharjah (2.13 in goats, Dubai[(G.D] vs 4.34 in goats, Sharjah [G.S]; two-sample paired t-test, $P$ $<0.01)$. The Index of Evenness was significantly higher in G.D than in G.S (0.344 vs 0.139 , respectively; two-sample

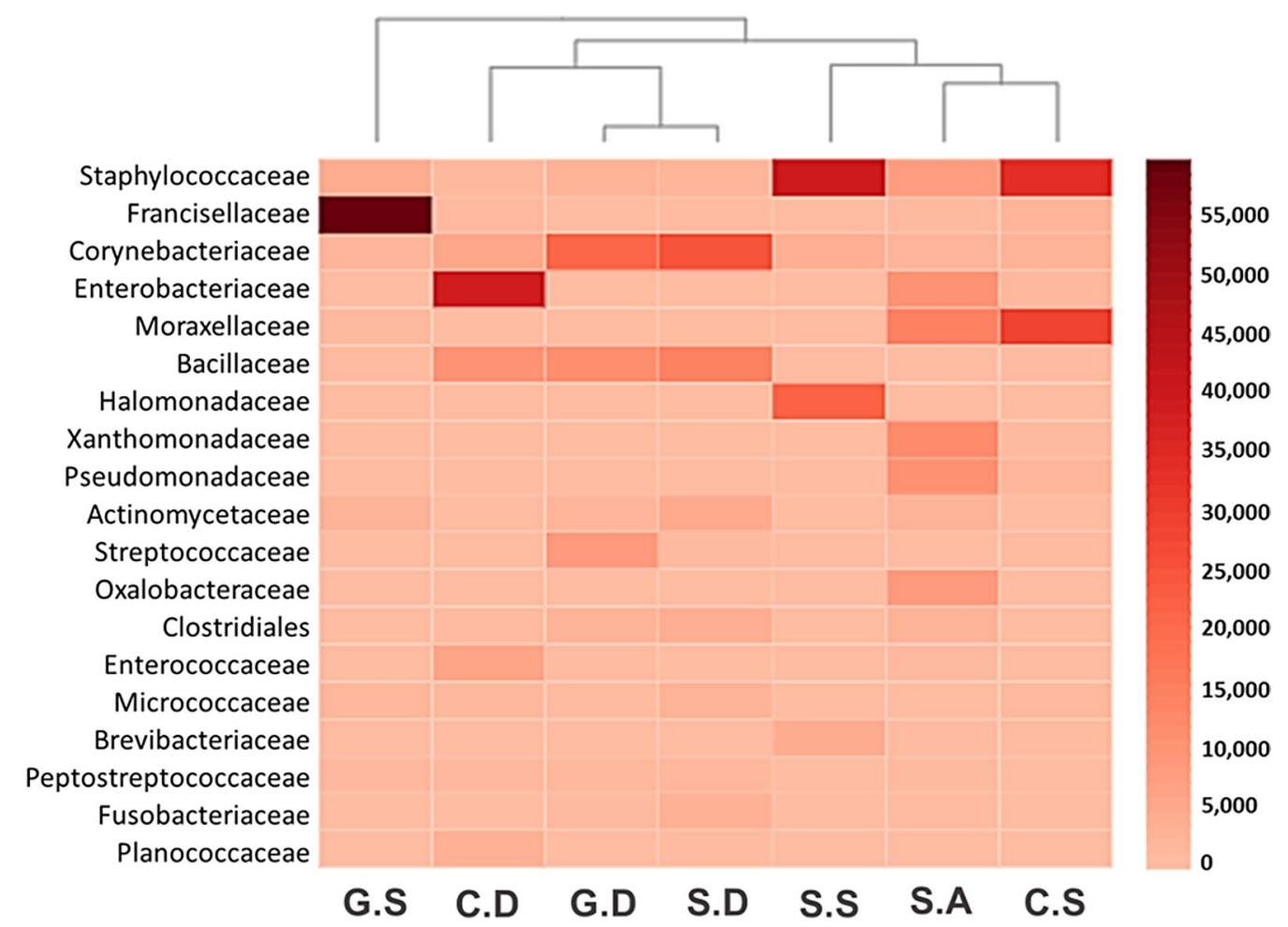

Fig. 1 Microbial families detected in H. anatolicum adult male ticks collected from goats, cows and sheep in three emirates of the United Arab Emirates in 2019 and 2020. Abbreviations: G.S, Goats, Sharjah; C.D, cows, Dubai; G.D, goats, Dubai; S.D, sheep, Dubai; S.S, sheep, Sharjah; S.A, sheep, Abu Dhabi; C.S, cows, Sharjah 


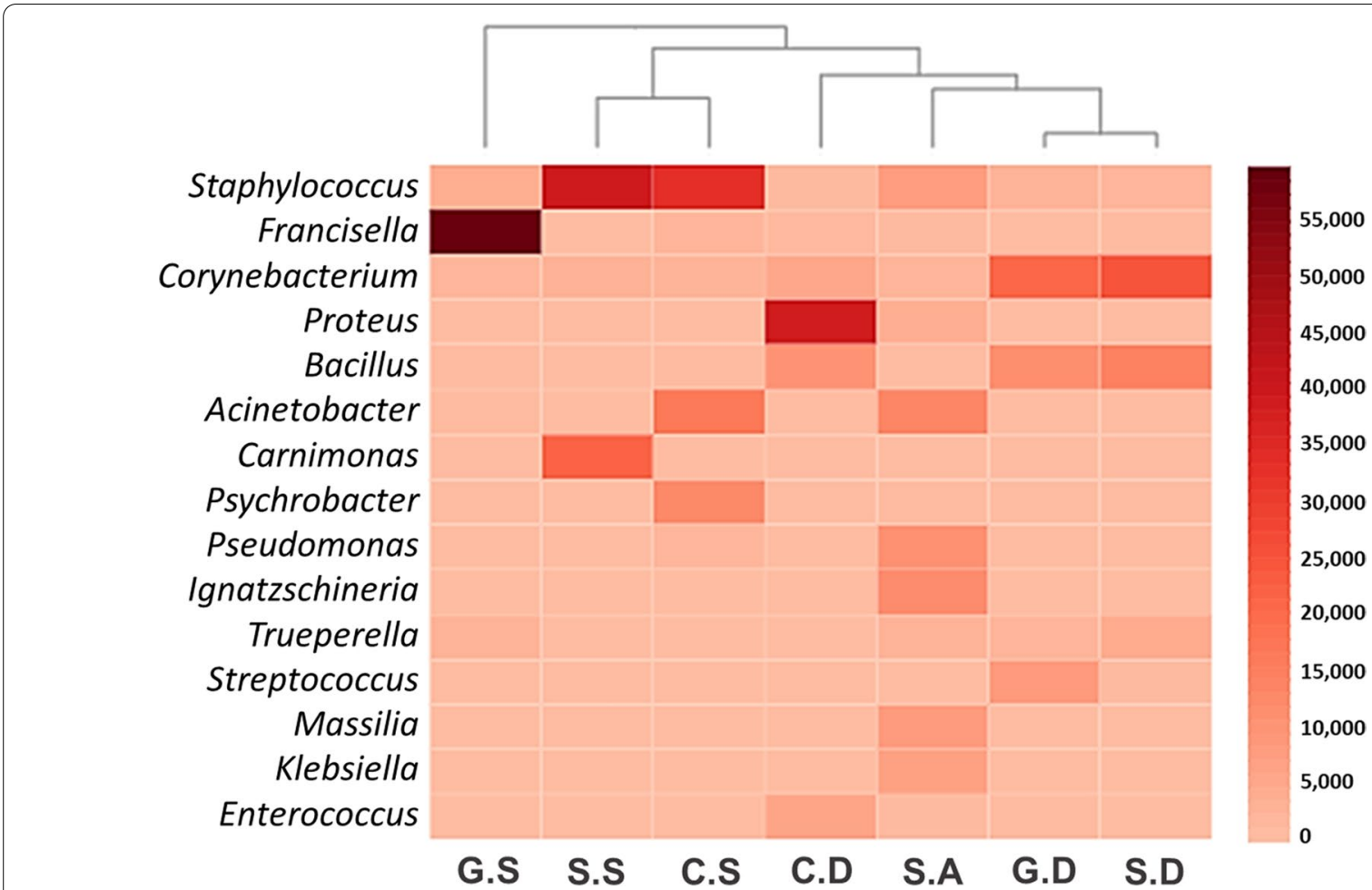

Fig. 2 Microbial genera detected in H. anatolicum adult male ticks collected from goats, cows and sheep in three emirates of the UAE in 2019 and 2020. Abbreviations as in Fig. 1 caption

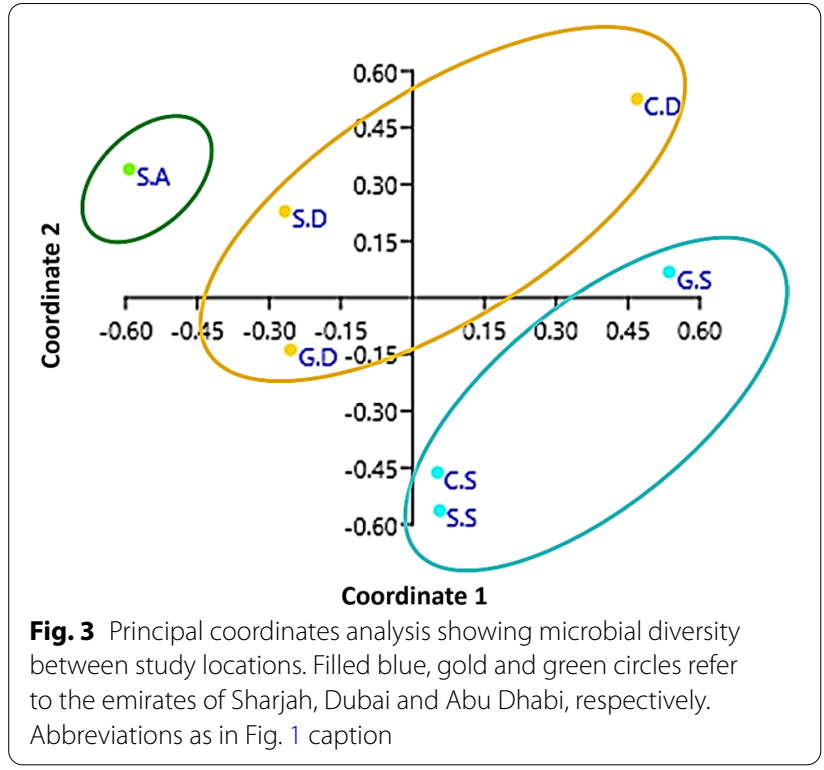

paired t-test, $P<0.01)$. The Index of Dominance was significantly different between G.D and G.S ( 0.32 vs 0.78 , respectively; two-sample paired t-test, $P<0.01)$. Further, the richness and evenness of genera were significantly different between sheep samples from Abu Dhabi, Dubai and Sharjah.

\section{Associations between bacterial genera}

Pearson's correlation coefficients $(r)$ indicated that several microbial genera were significantly correlated with each other $(P<0.05$, boxed circles) (Fig. 4; Additional file 6: Table S6). Fransicella was significantly positively correlated with Pseudomonas, Trueperella and Proteus; Acinetobacter and Enterococcus were positively correlated with some of the other genera. However, Klebsiella was negatively correlated with Carnimonas, Ignatzschineria, Pseudomonas, and Acinetobacter. In addition, Corynebacterium was negatively correlated with Carnimonas and Pseudomonas. We did not find significant relationships between response variables and explanatory variables.

\section{Discussion}

Overall, our results show that Staphylococcus, Francisella, Corynebacterium, Proteus and Bacillus were the most abundant microbial genera in $H$. anatolicum; other genera were also present but at lower levels. Thus, this study provides a snapshot of the internal microbiome and sheds light on the potential tick-borne pathogens and endosymbionts associated with $H$. anatolicum in the 

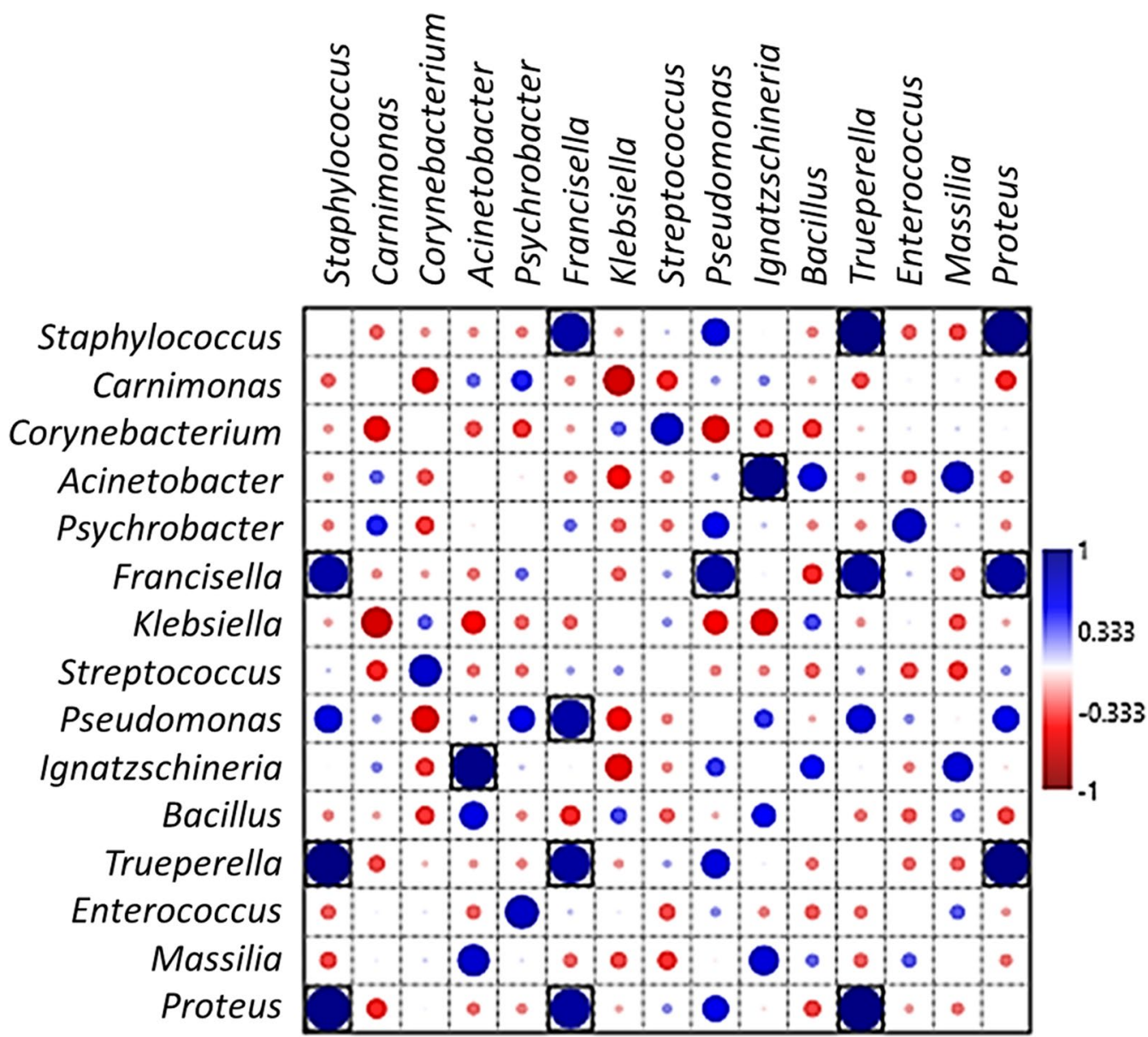

Fig. 4 Pearson's correlation coefficients indicating associations between bacterial genera showing significantly positive interactions (large dark-blue circles) and significantly negative interactions (large red circles). Black boxes denote $P<0.05$

UAE. Previous studies showed that vector-borne infections in the vertebrate host shape the microbiome of the arthropod vector and their competence to acquire and maintain infections with vector-borne pathogens [32]. Therefore, the results of the current study will help increase our understanding of disease dynamics in livestock. Our observation that Staphylococcus and Francisella were the most common genera in $H$. anatolicum indicates that some pathogenic species, such as Staphylococcus aureus or Francisella tularensis, may be present in the animal population this tick species parasitizes.

We obtained a lower number of bacterial reads and OTUs (372) from male $H$. anatolicum as compared to earlier studies which reported 1314 OTUs (sex of tick is not mentioned [6]) and 6023 OTUs (female $H$. anatolicum [33]) on the microbiome of $H$. anatolicum. This difference can probably be attributed to differences in the life-cycle due to the geographical separation of species and climatic conditions of the Middle East and Asia. Furthermore, our results support the observations from earlier Hyalomma tick microbiome studies regarding bacterial phyla. For example, the pattern of bacterial phyla presence in the current study is consistent with that reported in earlier studies in which Proteobacteria, Firmicutes and Actinobacteria were found to be abundant in $H$. anatolicum [6] and $H$. dromedarii [8]. In addition, we found Gammaproteobacteria and Bacilli to be dominant classes in $H$. anatolicum ticks collected from all three hosts, as reported earlier [19], however their relative abundance varied among the three sampling locations of the UAE.

In an earlier study we found that the bacterial family Francisellaceae was present in very high relative abundance (99.1\%) in the camel tick H. dromedarii [8]; in the present study we similarly detected Francisellaceae with high relative abundance $(72 \%)$ in $H$. anatolicum collected from goats (Fig. 1). In addition, we observed Enterobacteriaceae and Moraxellaceae to be present with high relative abundance (57.9\% and $38.8 \%$, respectively) in $H$. anatolicum collected from cows, which differs 
from the results of some other studies [19] that reported Staphylococcaceae, Oxalobacteraceae, Burkholderiaceae and Pseudomonades as dominant families in $H$. anatolicum collected from cattle. However, we detected a high relative abundance of Staphylococcaceae (57.7\%), Corynebacteriaceae (41.5\%), Halomonadaceae (31.7\%) and Bacillaceae (23.6\%) from sheep samples. The composition and diversity of bacterial families inside each individual tick were probably influenced by external and stochastic factors [8]. Further, tick microbial diversity could also be affected by host species and host species' competence to sustain different types of microbes.

In total, 153 genera were identified in H. anatolicum. Interestingly, Staphylococcus and Corynebacterium were present in almost all locations and, in addition, both genera were detected with high relative abundance (57.6\%) and $(41.5 \%)$ in ticks collected from sheep. Similar results have been reported previously, where Corynebacterium and Staphylococcus were the most dominant genera in tick species $[19,34]$; it is likely ticks acquire these microorganisms from livestock skin and fur. In the current study, Francisella was detected with a high relative abundance of $72 \%$ in ticks collected from goats, followed by Proteus (57.9\%) from cows. Recently, a high prevalence of endosymbionts, such as Francisella-like endosymbionts (91.5\%), has been reported in bovine ticks [18]; however, a previous study [19] reported Francisella with only $0.2 \%$ abundance in $H$. anatolicum ticks collected from buffaloes during a study exploring the microbiomes of ticks collected from different species of livestock from Pakistan. However, in the UAE, the genus Francisella was previously reported in $H$. dromedarii collected from camels, with a high relative abundance of $99.1 \%$ [8]. In addition, almost similar results were reported from Saudi Arabia, where Francisella was found to be a dominant genus with an abundance of 94.4\% [34] and 42.1\% [35] in H. dromedarii. It has been shown that tick endosymbionts mostly belong to the genera Francisella, Rickettsia and Coxiella [36] and that they probably have mutualistic relationships with ticks possibly also modulate tick vector capacity by influencing tick-borne pathogens' colonization and transmission to vertebrate hosts $[9,37]$. In addition, bacterial endosymbionts of ticks might influence survival, fitness and reproduction, nutritional adaptation and immunity $[9,13]$. The endosymbionts may vary significantly among tick species across the world, and their composition among tick species is affected by numerous factors, such as tick species $[38,39]$, tick developmental stage [40], feeding status of tick [41], co-existence of pathogens inside ticks [37], environmental conditions [41], seasonality [38], and geographical area [39]. Although the genera Francisella, Rickettsia and Coxiella might be present as endosymbionts in some tick species, including $H$. anatolicum, this does not rule out the possibility of the presence of some of their pathogenic species in a few tick populations now or in the future in the UAE. Therefore, this study underlines the importance of conducting disease screening programs to ensure the early detection of tick-borne pathogens. Additionally, our analysis indicated the presence of few read counts for the genus Ehrlichia in tick samples collected from cows from Dubai and Sharjah and goats from Dubai. However, Ghafar et al. [18] recently reported high abundance of Ehrlichia in $H$. anatolicum ticks collected from livestock. These differences might be linked to different factors between the habitats of the two studies. In general, mixed infections in ticks, such as Francisella-like endosymbionts and piroplasmids and/or Ehrlichia spp., may exist in ticks in the UAE. It should be mentioned that ticks which are co-infected with multiple pathogens might pose a serious threat to animals and humans, which may intensify the clinical complexity of diseases [42]. Thus, studies are required for large-scale PCR screening of pathogens, such as Ehrlichia, to assess their interaction with other pathogens/ endosymbionts as well as its zoonotic potential.

Overall, we found significant positive associations between Fransicella and Pseudomonas, as well as between Trueperella, and Proteus. Thus far, little is known about Francisella spp. and their associations with other microbes, and how they shape the tick microbiome and alter the tick-borne disease epidemiology. Studies have confirmed that symbiotic bacterial genera co-exist with pathogenic ones [12] and endosymbiotic forms under shared environmental preferences, could have significant patterns of positive or negative co-occurrence with pathogenic forms and could facilitate, limit or block pathogen transmission, depending on the nature of tick microbial interactions $[12,43]$. The genus Francisella has been reported in $H$. dromedarii in the UAE [8], and it likely to be found as an endosymbiont. However, globally, the phylogenetic similarities between endosymbiont and pathogenic forms suggest periodic and perhaps even frequent shifts from non-pathogenic to pathogenic forms [12]. Noteworthy, Trueperella species are opportunistic pathogens and considered to be a part of the biota of the skin and upper respiratory and urogenital tracts of cattle, goats and sheep; however, its reservoirs and transmission routes and pathogenesis are poorly understood [44]. In the present study, we found Trueperella with low abundance in goats and sheep samples (Fig. 2). In addition, we detected Pseudomonas in sheep, which is also an opportunistic pathogen that can be found in a variety of soils [45]. However, Brown et al. [46] found that adult Dermacentor andersoni ticks may be killed by Proteus mirabilis. Furthermore, we found a negative 
association between Klebsiella and Carnimonas, Ignatzschineria, Pseudomonas and Acinetobacter. Interestingly, Acinetobacter spp. occupy diverse environments, such as soil and freshwater, and many are known to be pathogenic, while others are considered to be commensal and part of the normal animal flora [47]. It is known that ticks can acquire microbiota of skin/ fur during feeding on the host, while the microbes present in the vegetation or soil probably colonize ticks on the ground when they drop off their vertebrate hosts [48]. However, tick-borne infections in the vertebrate host shape the microbiome of ticks [32]. We suggest that the five abovementioned bacterial genera need further confirmation through PCR screening using genusspecific primers. In addition, continuous surveillance and screening of bacterial genera is important for the development of mitigation strategies for tick-borne pathogens.

\section{Conclusions}

In this study, we quantified the internal microbiome present in male $H$. anatolicum. The findings of the current study will fuel interest to improve our understanding of the distribution pattern of microbes and to visualize the pathogens and symbionts in the microbial fauna of $H$. anatolicum and other livestock ticks in the UAE. Staphylococcus, Francisella, Corynebacterium, Proteus, and Bacillus were the most abundant microbial genera identified in this study. This work represents an initial step towards enhancing future comparative microbiome studies in the UAE and elsewhere. Nonetheless, it remains essential to mention that further research is required to investigate and screen samples using species-specific primers for the characterization of endosymbionts and tick-borne pathogens.

\section{Abbreviations}

C.D: Cows, Dubai; C.S: Cows, Sharjah; G.D: Goats, Dubai; G.S: Goats, Sharjah; S.A: Sheep, Abu Dhabi; S.D: Sheep, Dubai; S.S: Sheep, Sharjah; UAE: United Arab Emirates.

\section{Supplementary Information}

The online version contains supplementary material available at https://doi. org/10.1186/s13071-021-05144-z.

Additional file 1: Table S1. Read quality by sample.

Additional file 2: Table S2. Microbial phyla (presence in \%) detected in $\mathrm{H}$. anatolicum adult ticks from three emirates in the UAE.

Additional file 3: Table S3. Microbial classes (presence in \%) detected in $H$. anatolicum adult ticks from three emirates in the UAE.

Additional file 4: Table S4. Microbial families (presence in \%) detected in $H$. anatolicum adult ticks from three emirates in the UAE.
Additional file 5: Table S5. Microbial genera (presence in \%) detected in H. anatolicum adult ticks from three emirates in the UAE.

Additional file 6: Table S6. Correlation matrix showing pairwise Pearson's $r$ correlations between genera (bottom) and their associated significance (top).

\section{Acknowledgements}

We thank Amjad Saeed for his help in tick collection and the UAE University Transportation Department for providing vehicles for field work.

\section{Authors' contributions}

MAA and SBM conceived and designed the study. NP conducted the field work, processed the samples and conducted the experiments. NP, RV, SBM and MAA analyzed the data. NP and SBM performed the statistical analyses. NP and MAA prepared the graphs. NP, MAA, SBM and RV drafted the manuscript. MAA and SBM acquired funding. MAA supervised and managed the project. All authors read and approved the final manuscript.

Funding

The funding of this study was provided by the UAE University through UPAR Grant \# G00002604.

Availability of data and materials

All data generated or analyzed during this study are included in this published article.

\section{Declarations}

Ethics approval and consent to participate

Tick collection was carried out in strict accordance with the recommendations of the Animal Research Ethics Committee (A-REC) of UAE University (ethical approva no.: ERA_2019_5953). The experimental protocol was also approved by the UAE University Research Office.

\section{Consent for publication}

Not applicable.

\section{Competing interests}

All authors declare that they have no competing interests.

Received: 4 August 2021 Accepted: 27 December 2021

Published online: 20 January 2022

\section{References}

1. Vilenberg $G$. Veterinary significance of ticks and tick-borne diseases. In: Fivaz B, Petney T, Horak I, editors. Tick vector biology; Heidelberg: Springer; 1992. p. 23-33.

2. Perveen N, Muzaffar SB, Al-Deeb MA. Ticks and tick-borne diseases of livestock in the middle east and north Africa: a review. Insects. 2021;12:1-34.

3. El-Sayed A, Kamel M. Climatic changes and their role in emergence and re-emergence of diseases. Environ Sci Pollut Res. 2020;27:22336-52. https://doi.org/10.1007/s11356-020-08896-w.

4. Vatansever Z. Hyalomma anatolicum Koch, 1844. In: Estrada-Peña A, Mihalca AD, Petney TN, editors. Ticks Eur N Afr. Switzerland: Springer Cham; 2017;391-5.

5. Perveen N, Muzaffar SB, Al-Deeb MA. Four tick-borne microorganisms and their prevalence in Hyalomma ticks collected from livestock in United Arab Emirates. Pathogens. 2021;10:1005. https://doi.org/10.3390/ pathogens 10081005.

6. Adegoke A, Kumar D, Bobo C, Rashid MI, Durrani AZ, Sajid MS, et al. Tick-borne pathogens shape the native microbiome within tick vectors. Microorganisms. 2020;8:1-16.

7. Lejal E, Chiquet J, Aubert J, Robin S, Estrada-Peña A, Rue O, et al. Temporal patterns in /xodes ricinus microbial communities: an insight into tickborne microbe interactions. Microbiome. 2020;9:1-20. 
8. Perveen N, Muzaffar SB, Vijayan R, Al-Deeb MA. Microbial communities associated with the camel tick, Hyalomma dromedarii: 16S rRNA genebased analysis. Sci Rep. 2020;10:1-11.

9. Wu-chuang A, Hodžić A, Mateos-hernández L, Estrada-peña A, Obregon D, Cabezas-cruz A. Current debates and advances in tick microbiome research. Curr Res Parasitol Vector-Borne Dis. 2021. https://doi.org/10. 1016/j.crpvbd.2021.100036.

10. Narasimhan S, Rajeevan N, Liu L, Zhao YO, Heisig J, Pan J, et al. Gut microbiota of the tick vector Ixodes scapularis modulate colonization of the Lyme disease spirochete. Cell Host Microbe. 2014;15:58-71. https://doi. org/10.1016/j.chom.2013.12.001.

11. Gall CA, Reif KE, Scoles GA, Mason KL, Mousel M, Noh SM, et al. The bacterial microbiome of Dermacentor andersoni ticks influences pathogen susceptibility. ISME J. 2016;10:1846-55.

12. Bonnet SI, Binetruy F, Hernández-jarguín AM, Duron O. The tick microbiome: why non-pathogenic microorganisms matter in tick biology and pathogen transmission. Front Cell Infect Microbiol. 2017;7:1-14.

13. Bonnet SI, Pollet T. Update on the intricate tango between tick microbiomes and tick-borne pathogens. Parasite Immunol. 2021;43:1-12.

14. Smith TA, Driscoll T, Gillespie JJ, Raghavan R. A Coxiella-like endosymbiontis a potential vitamin source for the lone star tick. Genome Biol Evol. 2015;7:831-8

15. Jasinskas A, Zhong J, Barbour AG. Highly prevalent Coxiella sp. bacterium in the tick vector Amblyomma americanum. Appl Environ Microbiol. 2007;73:334-6.

16. Klyachko O, Stein BD, Grindle N, Clay K, Fuqua C. Localization and visualization of a Coxiella-type symbiont within the lone star tick Amblyomma americanum. Appl Environ Microbiol. 2007;73:6584-94.

17. Zhong J, Jasinskas A, Barbour AG. Antibiotic treatment of the tick vector Amblyomma americanum reduced reproductive fitness. PLOS ONE. 2007:2:405.

18. Ghafar A, Cabezas-Cruz A, Galon C, Obregon D, Gasser RB, Moutailler S, et al. Bovine ticks harbour a diverse array of microorganisms in Pakistan. Parasit Vectors. 2020;13:1-15. https://doi.org/10.1186/s13071-019-3862-4.

19. Karim S, Budachetri K, Mukherjee N, Williams J, Kausar A, Hassan MJ, et al. A study of ticks and tick-borne livestock pathogens in Pakistan. PLoS Negl Trop Dis. 2017;11:1-17.

20. Perveen N, Muzaffar SB, Al-Deeb MA. Population dynamics of Hyalomma dromedarii on camels in the United Arab Emirates. Insects. 2020;11:1-9.

21. Apanaskevich DA. Differentiation of closely related species Hyalomma anatolicum and Hyalomma excavatum (Acari, Ixodidae) based on a study of all life cycle stages, throughout entire geographical range. Parazitologiia. 2003;37:259-80.

22. Walker AR, Bouattour A, Camicas JL, Estrada-Peña A, Horak IG, Latif AA et al. Ticks of domestic animals in Africa: a guide to identification of species. Edinburgh: Bioscience Reports; 2003. http://www.alanrwalker.com/ assets/PDF/tickguide-africa.pdf.

23. Carpi G, Cagnacci F, Wittekindt NE, Zhao F, Qi J, Lynn P, et al. Metagenomic profile of the bacterial communities associated with Ixodes ricinus ticks. PLoS ONE. 2011;6:e25604.

24. Klindworth A, Pruesse E, Schweer T, Peplies J, Quast C, Horn M, et al. Evaluation of general 165 ribosomal RNA gene PCR primers for classical and next-generation sequencing-based diversity studies. Nucleic Acids Res. 2013;41:1-11.

25. Magoč T, Salzberg SL. FLASH: fast length adjustment of short reads to improve genome assemblies. Bioinformatics. 2011;27:2957-63.

26. Li W, Fu L, Niu B, Wu S, Wooley J. Ultrafast clustering algorithms for metagenomic sequence analysis. Brief Bioinform. 2012;13:656-68.

27. Caporaso JG, Kuczynski J, Stombaugh J, Bittinger K, Bushman FD, Costello EK, et al. QIIME allows analysis of high-throughput community sequencing data. Nat Methods. 2010;7:335.

28. Altschul SF, Gish W, Miller W, Myers EW, Lipman DJ. Basic local alignment search tool. J Mol Biol. 1990;215:403.

29. Hammer $\varnothing$, Harper DAT, Ryan PD. PAST: Paleontological Statistics Software Package for education and data analysis. Palaeontol Electron. 2001;4:1-9.

30. Paliy O, Shankar V. Application of multivariate statistical techniques in microbial ecology. Mol Ecol. 2017;25:1032-57.

31. Sokal RR, Rohlf FJ. Biometry: the principles and practice of statistics in biological. 4th edition. New York:W.H. Freeman and Co.; 2012. https://doi. org/10.2307/2343822
32. Hamilton PT, Maluenda E, Sarr A, Belli A, Hurry G, Duron O, et al. Borrelia afzelii infection in the rodent host has dramatic effects on the bacterial microbiome of Ixodes ricinus ticks. Appl Environ Microbiol. 2021;87:e00641-21.

33. Choubdar N, Karimian F, Koosha M, Oshaghi MA. An integrated overview of the bacterial flora composition of Hyalomma anatolicum, the main vector of cchf. PLoS Negl Trop Dis. 2021;15:1-15. https://doi.org/10.1371/ journal.pntd.0009480.

34. Elbir H, Almathen F, Alhumam NA. A glimpse of the bacteriome of Hyalomma dromedarii ticks infesting camels reveals human Helicobacter pylori pathogen. J Infect Dev Ctries. 2019;13:1001-12.

35. Alreshidi MM. Description of microbial diversity associated with ticks Hyalomma dromedarii (Acari: Ixodidae) isolated from camels in Hail region (Saudi Arabia) using massive sequencing of 165 rDNA. Bioinformation. 2020;16:602-10.

36. Ahantarig A, Trinachartvanit W, Baimai V, Grubhoffer L. Hard ticks and their bacterial endosymbionts (or would be pathogens). Folia Microbiol (Praha). 2013;58:419-28.

37. Abraham NM, Liu L, Jutras BL, Yadav AK, Narasimhan S, Gopalakrishnan $\checkmark$, et al. Pathogen-mediated manipulation of arthropod microbiota to promote infection. Proc Natl Acad Sci USA. 2017;114:E781-90. https://doi. org/10.1073/pnas.1613422114.

38. Lalzar I, Harrus S, Mumcuoglu KY, Gottlieb Y. Composition and seasonal variation of Rhipicephalus turanicus and Rhipicephalus sanguineus bacterial communities. Appl Environ Microbiol. 2012;78:4110-6.

39. van Treuren W, Ponnusamy L, Brinkerhoff RJ, Gonzalez A, Parobek CM, Juliano JJ, et al. Variation in the microbiota of Ixodes ticks with regard to geography, species, and sex. Appl Environ Microbiol. 2015;81:6200-9.

40. Moreno CX, Moy F, Daniels TJ, Godfrey HP, Cabello FC. Molecular analysis of microbial communities identified in different developmental stages of Ixodes scapularis ticks from Westchester and Dutchess Counties, New York. Environ Microbiol. 2006;8:761-72.

41. Heise SR, Elshahed MS, Little SE. Bacterial diversity in Amblyomma americanum (Acari: Ixodidae) with a focus on members of the genus Rickettsia. J Med Entomol. 2010;47:258-68.

42. Wikel SK. Ticks and tick-borne infections: complex ecology, agents, and host interactions. Vet Sci. 2018;5:60.

43. Aivelo T, Norberg A, Tschirren B. Bacterial microbiota composition of Ixodes ricinus ticks: the role of environmental variation, tick characteristics and microbial interactions. PeerJ. 2019;7:e8217.

44. Rzewuska M, Kwiecien E, Chrobak-Chmiel D, Kizerwetter-Swida M, Stefanska I, Gierynska M. Pathogenicity and virulence of Trueperella pyogenes: a review. Int J Mol Sci. 2019;20:1-33.

45. Zimmer KR, Macedo AJ, Nicastro GG, Baldini RL, Termignoni C. Egg wax from the cattle tick Rhipicephalus (Boophilus) microplus inhibits Pseudomonas aeruginosa biofilm. Ticks Tick-borne Dis. 2013;4:366-76. https:// doi.org/10.1016/j.ttbdis.2013.01.005.

46. Brown RS, Reichelderfer CF, Anderson WR. An endemic disease among laboratory populations of Dermacentor andersoni ( $=$ D. venustus) (Acarina: Ixodidae). J Invertebr Pathol. 1970;16:142-3.

47. van der Kolk JH, Endimiani A, Graubner C, Gerber V, Perreten V. Acinetobacter in veterinary medicine, with an emphasis on Acinetobacter baumannii. J Glob Antimicrob Resist. 2019;16:59-71. https://doi.org/10. 1016/j.jgar.2018.08.011.

48. Narasimhan S, Fikrig E. Tick microbiome: the force within. Trends Parasitol. 2015. https://doi.org/10.1016/j.pt.2015.03.010.

\section{Publisher's Note}

Springer Nature remains neutral with regard to jurisdictional claims in published maps and institutional affiliations. 